\title{
Casimir effect between anti-de Sitter braneworlds
}

\author{
James P. Norman* \\ School of Mathematics and Statistics, University of Newcastle upon Tyne, \\ Newcastle upon Tyne, NE1 7RU, United Kingdom
}

(Dated: 30 June 2004)

\begin{abstract}
We calculate the one-loop effective action of a scalar field with general mass and coupling to the curvature in the detuned Randall-Sundrum brane world scenario, where the four-dimensional branes are anti-de Sitter. We make use of conformal transformations to map the problem to one on the direct product of the hyperbolic space $\mathrm{H}^{4}$ and the interval. We also include the cocycle function for this transformation. This Casimir potential is shown to give a sizable correction to the classical radion potential for small values of brane separation.
\end{abstract}

PACS numbers: 11.10.Kk, 04.62.+v, 04.50.+h

\section{INTRODUCTION}

The one- and two- brane Randall-Sundrum models [1, 2] have become one of the most popular scenarios for the discussion of brane world cosmology. The two-brane model was proposed as a solution to the the hierarchy problem without introducing supersymmetry. In the original two-brane Randall-Sundrum model, the brane tensions are tuned so that the branes are flat. However, solutions also exist when the brane tensions are detuned, with either $d S_{4}$ or $A d S_{4}$ branes $3,[4]$.

The de Sitter case has been looked at as a model for inflation [5]. The anti-de Sitter brane case is relevant to a supersymmetric extension of the Randall-Sundrum model which is based on gauged five-dimensional supergravity [6, 7, 8]. In this model, a locally supersymmetric coupling of five dimensional gauged supergravity to branes requires that the branes are $A d S_{4}$ or $M_{4}$.

Upon compactification to four dimensions, the separation of the branes is promoted to a field, called the radion. In case where the branes are flat, the classical potential for the radion is zero. This would lead to a massless scalar particle, which is not seen in nature. This is the radion stabilisation problem. One possible solution is that the quantum vacuum energy of the bulk fields could generate a potential, analogous to the Casimir effect, to stabilise of the radion. This mechanism has been looked at in 9 , 10, 11, 12, 13, 14] for bulk scalar fields, and [15, 16] for fermions, where it was found that it is not possible to simultaneously solve the hierarchy problem and have an acceptable mass for the radion. A related calculation in five dimensional heterotic $M$ theory has been performed in [17, 18].

When the branes are $A d S_{4}$ or $d S_{4}$, the brane separation is fixed by the junction conditions and there is a classical potential for the radion [19]. The potential is a stabilizing one in the $A d S_{4}$ case, and an unstable one in the $d S_{4}$ case. The Casimir effect would then be a quantum correction to the classical potential. In the $d S_{4}$ brane case, the quantum effective potential has been calculated for conformally coupled scalar fields in [20] and for massless fermions in [21]. Small deviations from the conformal values were considered in [22].

The Casimir effect between two anti-de Sitter branes has not yet received much attention. This may be because the model is not as relevant to cosmology as its de Sitter cousin. However, there seems to be no a priori reason to favour the $d S_{4}$ case over the $A d S_{4}$ case, since the brane tensions are put in "by hand". The Casimir effect in the supersymmetric $A d S_{4}$ case may be important as it may give a dynamical mechanism for breaking supersymmetry (see 23] for a related calculation on a flat orbifold). However, the calculation of the effective action of the higher spin fields in the supergravity multiplet in this background presents a difficult technical challenge. To simplify the calculation and illustrate our method, here we will consider only a scalar field in the background with $A d S_{4}$ branes, with the hope that many of the features of the calculation apply to higher spin fields. Some related work on the one-loop effective potential of $A d S_{n} \times S^{n}$ was performed in 24]. Spontaneous generation of $A d S_{4}$ branes in an $A d S_{5}$ bulk has been investigated in [25, 26].

We calculate the one-loop effective action for scalar fields with general mass and coupling to the scalar curvature. Both Dirichlet and Robin type boundary conditions will be considered. It will be shown that the quantum effective potential can give a sizable correction to the classical potential for small values of the radion. Our calculation cannot be carried over easily to the $d S_{4}$ case, because of the different global properties of $d S_{4}$ and $A d S_{4}$.

*Electronic address: j.p.norman@ncl.ac.uk 
We choose to work in the "downstairs" picture of a manifold with boundary and impose boundary conditions on the fields, rather than the "upstairs" picture of an orbifold with singular branes. The one-loop effective action of the scalar field is evaluated using $\zeta$ function regularization. We use the properties of the effective action under conformal rescalings of the metric to relate the effective action to that of a scalar field on the direct product of $A d S_{4} \times I$. $\zeta$ function regularization requires that we rotate to the appropriate Euclidean space, which is $\mathrm{H}^{4} \times I$, where $\mathrm{H}^{4}$ is the four dimensional hyperbolic space. We then calculate the correction factor for this transformation, known as the cocycle function. Our curvature conventions are given in [18].

\section{OPERATORS AND BACKGROUND METRIC}

The background metric for the Randall-Sundrum model with $A d S_{4}$ branes can be written as

$$
d s^{2}=e^{-2 \omega(z)}\left(g_{\mu \nu}^{(4)} d x^{\mu} d x^{\nu}+d z^{2}\right)
$$

where $g_{\mu \nu}^{(4)}$ is the metric of $A d S_{4}$ with $A d S$ radius $a$,

$$
\omega(z)=\ln \left(\sqrt{\frac{|\Lambda|}{6}} a \sin (z / a)\right),
$$

and $\Lambda$ is the bulk cosmological constant. In the absence of branes, the conformal coordinate $z$ would run from $0<z<a \pi$. However, in the "downstairs" picture of a manifold with boundaries, the branes cut off the space and $z$ is restricted to the space between the branes. The positions of the branes are fixed at the classical level by junction conditions on the metric. We call the positions of the branes $z_{1}$ and $z_{2}$. Explicitly, the classical positions of the branes are

$$
z_{1,2}^{\text {classical }}=a \arccos \frac{\sigma_{1,2}}{\sigma}
$$

where $\sigma=\sqrt{|\Lambda| / 6}, \sigma_{1}=T_{1} / 6, \sigma_{2}=-T_{2} / 6$, and $T_{1,2}$ are the tensions of the branes at $z_{1}$ and $z_{2}$, respectively. However, these values may receive corrections from quantum effects, so we keep $z_{1}$ and $z_{2}$ general. One condition we can always impose is that the metric on the brane at $z_{1}$ has a scale factor of unity. This fixes $\sin \left(z_{1} / a\right)=(\sigma a)^{-1}$. Without loss of generality, we also restrict $z_{2}>z_{1}$.

In the limit $a \rightarrow \infty$, the function $w(z) \approx \ln (\sigma z)$, and we recover the flat brane Randall-Sundrum metric. In this limit, the positions of the branes are no longer determined by the junction conditions, with all brane positions being allowed.

We consider the effective action of a scalar field $\phi$ with general mass $m$ and coupling to the scalar curvature $R$. The fluctuation operator is

$$
\Delta=-\nabla^{2}+\xi R+m^{2}
$$

The one-loop effective action will be regularized using $\zeta$-function regularization. We work in Euclidean space, with $A d S_{4}$ becoming the hyperbolic space $\mathrm{H}^{4}$. The $\zeta$ function is defined as the trace of the operator $\Delta$ to some power $-s$, in some region of the complex plane where the trace converges, i.e.,

$$
\zeta(s)=\operatorname{tr} \Delta^{-s}=\sum_{n} \int_{0}^{\infty} d \lambda \mu(\lambda) d_{n} \rho_{n}(\lambda)^{-s} .
$$

Here, the eigenvalues of the operator $\rho_{n}(\lambda)$ are assumed to split into a continuous part, labeled by the real parameter $\lambda$, and a discrete part, labeled by integers $n$. The spectral function $\mu(\lambda)$ gives a "density of states" in the continuous spectrum, and is analogous to the discrete degeneracy factor $d_{n}$. The one loop effective action is then defined to be

$$
W=-\frac{1}{2} \zeta^{\prime}(0)-\frac{1}{2} \zeta(0) \log \mu_{R}^{2},
$$

where we have analytically continued the $\zeta$ function to $s=0$. The renormalisation scale $\mu_{R}$ has been introduced to make the eigenvalues dimensionless. We can also define the effective potential on the brane at $z_{1}$ by dividing throughout by the volume of the $\mathrm{H}^{4}$ space. That is, we define the one-loop effective potential $V$ through

$$
W=\int\left|g^{(4)}\right| d^{4} x V .
$$


Rather than work directly with the eigenvalues of Eq. (4), which are difficult to obtain in curved space, we can use the behaviour of the effective action under conformal rescalings of the metric to simplify the problem. Considering operators of Laplace type, with $\Delta=-\nabla^{2}+X$, we introduce a one-parameter family of metrics $g^{\epsilon}$ related to the physical metric by a conformal rescaling, so that

$$
g_{\alpha \beta}^{\epsilon}=\Omega(\epsilon)^{2} g_{\alpha \beta}, \quad \Omega(\epsilon)=e^{(1-\epsilon) \omega(z)} .
$$

The conformally rescaled operator is $\Delta_{\epsilon}=-\nabla_{\epsilon}^{2}+X_{\epsilon}$, where $X_{\epsilon}=X \Omega(\epsilon)^{-2}-\frac{3}{16}\left(R \Omega(\epsilon)^{-2}-R_{\epsilon}\right)$. One can then show that [27]

$$
W[\epsilon=1, \Delta]=W\left[\epsilon=0, \Delta_{0}\right]+C[\Omega],
$$

where the cocycle function $C[\Omega]$ is given (in five dimensions) in terms of the generalized heat kernel coefficient $B_{5 / 2}(f, \Delta)$ as

$$
C[\Omega]=\int_{0}^{1} d \epsilon B_{5 / 2}\left(\omega, \Delta_{\epsilon}\right)
$$

Hence, we can relate the one-loop effective action of the scalar field in the warped metric (11) to one on the direct product manifold $\mathrm{H}^{4} \times I$.

We will first consider the effective action of the conformally transformed operator. The conformally transformed operator $\Delta_{0}$ separates into

$$
\Delta_{0}=\Delta_{I}+\Delta^{(4)}
$$

where $\Delta_{I}$ contains all dependence on the $z$-direction

$$
\Delta_{I}=-\partial_{z}^{2}-\xi \frac{12}{a^{2}}+\left(\xi-\frac{3}{16}\right)\left(8 \omega^{\prime \prime}-12 \omega^{2}\right)+m^{2} e^{-2 \omega}
$$

and $\Delta^{(4)}$ is the 4-dimensional Laplacian for a massless scalar field on $\mathrm{H}^{4}$. The eigenvalues and $\zeta$ function for $\Delta^{(4)}$ have been calculated by Camporesi [28]. The eigenvalues are continuous and labeled by the real parameter $\lambda$. The eigenvalues of $\Delta_{I}$, which we denote $m_{n}^{2}$, are discrete. After performing a separation of variables, we can regard the complete $\zeta$-function as a sum over $\zeta$-functions of scalar fields with mass $m_{n}$ on $\mathrm{H}^{4}$ [36]. Thus, from [28], we find

$$
\begin{aligned}
\zeta(s)=\frac{a^{2 s-4}}{8 \pi^{2}} \int\left|g^{(4)}\right|^{1 / 2} \sum_{n} & \left\{\frac{b_{n}^{2-2 s}}{8(s-1)}+\frac{b_{n}^{4-2 s}}{2(s-1)(s-2)}\right. \\
& \left.-2 \int_{0}^{\infty} d \lambda \frac{\lambda\left(\lambda^{2}+\frac{1}{4}\right)}{\left(1+e^{2 \pi \lambda}\right)\left(\lambda^{2}+b_{n}^{2}\right)^{s}}\right\},
\end{aligned}
$$

where we must restrict $s>5 / 2$, and we have introduced $b_{n}^{2}=\frac{9}{4}+m_{n}^{2} a^{2}$.

The eigenvalues $m_{n}^{2}$ of the operator $\Delta_{I}$ now need to be found. We consider both Dirichlet and Robin boundary conditions on the field at the boundaries $z_{1}$ and $z_{2}$. However, we shall illustrate our method using Dirichlet boundary conditions, and simply quote results for Robin boundary conditions in Appendix A as the calculation is similar. The eigenfunctions of Eq. (12) can be written in terms of associated Legendre functions of order $\mu=\sqrt{4-20 \xi+m^{2} / \sigma^{2}}$ and degree $-1 / 2+b_{n}$. For later convenience, we define the functions

$$
\begin{aligned}
& R_{-1 / 2+b_{n}}^{-\mu}(\theta)=\sqrt{\sin \theta}\left(\mathrm{P}_{-1 / 2+b_{n}}^{-\mu}(\cos \theta)\right), \\
& S_{-1 / 2+b_{n}}^{\mu}(\theta)=\sqrt{\sin \theta}\left(\mathrm{P}_{-1 / 2+b_{n}}^{\mu}(\cos \theta)-\frac{2 i}{\pi} \mathrm{Q}_{-1 / 2+b_{n}}^{\mu}(\cos \theta)\right) .
\end{aligned}
$$

These functions are linearly independent for all $\mu$ and $b_{n}$. The general solution to $\Delta_{I} f_{n}=m_{n}^{2} f_{n}$ is then a linear combination of $R_{-1 / 2+b_{n}}^{-\mu}(z / a)$ and $S_{-1 / 2+b_{n}}^{\mu}(z / a)$. Applying Dirichlet boundary conditions $\phi=0$ on the boundaries $\partial \mathcal{M}$, leads to an implicit equation for $b_{n}$ through

$$
F\left(b_{n}\right)=R_{-1 / 2+b_{n}}^{-\mu}\left(\theta_{1}\right) S_{-1 / 2+b_{n}}^{\mu}\left(\theta_{2}\right)-R_{-1 / 2+b_{n}}^{-\mu}\left(\theta_{2}\right) S_{-1 / 2+b_{n}}^{\mu}\left(\theta_{1}\right)=0,
$$

where $\theta_{1,2}=z_{1,2} / a$. 


\section{ZETA FUNCTION ON $\mathrm{H}^{4} \times I$}

The sums over the discrete eigenvalues $b_{n}$ in Eq. (13) are complicated by the fact that we only know the $b_{n}$ through the implicit equation $F\left(b_{n}\right)=0$. However, we can use techniques developed for studying the Casimir effect on balls and spheres 29, 30 to convert the sums into contour integrals of the function $F$. We will first consider the terms $\sum_{n} b_{n}^{2-2 s}$ and $\sum_{n} b_{n}^{4-2 s}$ in the $\zeta$ function. Our procedure follows that of the flat brane case, in that we write the sum as

$$
\hat{\zeta}(s)=\sum_{n} b_{n}^{-s}=\frac{1}{2 \pi i} \int_{\mathcal{C}} d z z^{-s} \frac{F^{\prime}(z)}{F(z)}
$$

where the contour $\mathcal{C}$ encloses all the real positive roots of $F(z)=0$. As in the flat brane case, we wish to write this contour integral as an integral over the imaginary axis. To do this, we need to know the asymptotic behaviour of $F(i x)$ for large $x$. We define the functions $\Sigma^{R}(x, \theta)$ and $\Sigma^{S}(x, \theta)$ by

$$
R_{-1 / 2+i x}^{-\mu}(\theta)=e^{i \pi \mu / 2+i \pi / 4} \sqrt{\frac{1}{2 \pi}} e^{x \theta} \frac{\Gamma(i x-\mu+1 / 2)}{\Gamma(i x+1)} \Sigma^{R}(x, \theta)
$$

and

$$
S_{-1 / 2+i x}^{\mu}(\theta)=e^{i \pi \mu / 2-i \pi / 4} \sqrt{\frac{2}{\pi}} e^{-x \theta} \frac{\Gamma(i x+\mu+1 / 2)}{\Gamma(i x+1)} \Sigma^{S}(x, \theta) .
$$

From the representation of the Legendre functions in terms of hypergeometric series 31, page 146], it can be seen that $\Sigma^{R}(x, \theta)$ and $\Sigma^{S}(x, \theta)$ have asymptotic series of the Poincaré type - that is, asymptotic expansions in inverse powers of $x$. We can also use properties of the $\Gamma$ function [32, page 256] to show

$$
\ln \left|\frac{\Gamma(i x+\mu+1 / 2) \Gamma(i x-\mu+1 / 2)}{\Gamma(i x+1) \Gamma(i x+1)}\right|=\ln \left|\frac{\sinh (\pi x)}{x \cosh [\pi(x-i \mu)]}\right| \sim-\ln (x)+O\left(e^{-x}\right) .
$$

Hence, for large $x$,

$$
\ln |F(i x)| \sim\left(\theta_{2}-\theta_{1}\right) x+\chi \ln (x)-\ln \pi+O\left(x^{-2}\right)
$$

where

$$
\chi=-1
$$

Therefore, for $s>1$, the contour can be deformed to an integral over the imaginary axis. After a few manipulations, we find

$$
\begin{aligned}
\hat{\zeta}(s)= & \frac{\sin \frac{\pi s}{2}}{\pi} \int_{\varepsilon}^{\infty} d x x^{-s} \frac{d}{d x} \ln \left|\frac{F(i x)}{F_{a}(i x)}\right|+\frac{\sin \frac{\pi s}{2}}{\pi} \int_{\varepsilon}^{\infty} d x x^{-s} \frac{d}{d x} \ln \left|F_{a}(i x)\right| \\
& +\frac{1}{2 \pi i} \int_{\mathcal{C}_{\varepsilon}} d z z^{-s} \frac{F^{\prime}(z)}{F(z)}
\end{aligned}
$$

where $\mathcal{C}_{\varepsilon}$ is a small semicircle of radius $\varepsilon$ around the origin, and

$$
F_{a}(i x)=R_{-1 / 2+i x}^{-\mu}\left(\theta_{2}\right) S_{-1 / 2+i x}^{\mu}\left(\theta_{1}\right)
$$

The first term on the RHS of Eq. (22) can now be continued to $s=-2$ and $s=-4$. The second term still cannot be analytically continued to these points as the integrals diverge at large $x$ if we take $s<1$. We therefore add and subtract terms which cause the integral to diverge in this region to enable us to analytically continue this term to $s=-2 N$, where $N$ is a positive integer. If we define $r_{k}$ and $s_{k}$ as the coefficients in the asymptotic expansion of $\ln \left|\Sigma^{R}(x, \theta)\right|$ and $\ln \left|\Sigma^{S}(x, \theta)\right|$, i.e.,

$$
\ln \left|\Sigma^{R}(x, \theta)\right| \sim \sum_{k=1}^{\infty} r_{k}(\theta) x^{-k}+O\left(e^{-x}\right), \quad \ln \left|\Sigma^{S}(x, \theta)\right| \sim \sum_{k=1}^{\infty} s_{k}(\theta) x^{-k}
$$


then we can define

$$
\begin{aligned}
\ln \left|F_{a}(i x)\right|_{\mathrm{reg}}= & \ln \left|F_{a}(i x)\right|+\ln \pi-\left(\theta_{2}-\theta_{1}\right) x-\chi \ln (x) \\
& -\sum_{k=1}^{2 N-1}\left(r_{k}\left(\theta_{1}\right)+s_{k}\left(\theta_{2}\right)\right) x^{-k}-\left(r_{2 N}\left(\theta_{2}\right)+s_{2 N}\left(\theta_{1}\right)\right) x^{-2 N} e^{-1 / x} .
\end{aligned}
$$

so that $\ln \left|F_{a}(i x)\right|_{\text {reg }} \sim O\left(x^{-2 N-1}\right)$ for large $x$. We can now substitute the for $\ln \left|F_{a}(i x)\right|$ in terms of $\ln \left|F_{a}(i x)\right|_{\text {reg }}$ in Eq. (22), and analytically perform the integrals of the "extra" terms in the right-hand side of Eq. (25). We can now analytically continue to $s=-2 N$. Provided that $F(x)$ is finite as $x \rightarrow 0$, the contribution from the integral around the small semicircle $\mathcal{C}_{\varepsilon}$ vanishes when we take $\varepsilon \rightarrow 0$. Thus, we find

$$
\hat{\zeta}(-2 N)=-(-1)^{N} N\left(r_{2 N}\left(\theta_{2}\right)+s_{2 N}\left(\theta_{1}\right)\right),
$$

and

$$
\begin{aligned}
\hat{\zeta}^{\prime}(-2 N)=-N(-1)^{N} & \left\{\int_{0}^{\infty} d x x^{2 N-1} \ln \left|\frac{F(i x)}{F_{a}(i x)}\right|+\int_{0}^{\infty} d x x^{2 N-1} \ln \left|F_{a}(i x)\right|_{\text {reg }}\right. \\
& \left.-\left(\gamma+\frac{1}{2 N}\right)\left(r_{2 N}\left(\theta_{1}\right)+s_{2 N}\left(\theta_{2}\right)\right)\right\} .
\end{aligned}
$$

where $\gamma$ is Euler's constant. We will quote the value of the first four coefficients $r_{k}$ and $s_{k}$ in Appendix B

For the third term in Eq. (13), we can interchange the order of integration and summation for large $s$. The resulting sum is not of the form considered above, but is a generalized Epstein type $\zeta$ function. Following Bordag et al. [30], we can again write the sum as a contour integral of $F$ around a contour which encloses all the positive zeros of $F$.

$$
\tilde{\zeta}(s)=\sum_{n}\left(\lambda^{2}+b_{n}^{2}\right)^{-s}=\frac{1}{2 \pi i} \int_{\mathcal{C}} d z\left(\lambda^{2}+z^{2}\right)^{-s} \frac{F^{\prime}(z)}{F(z)} .
$$

For $1 / 2<s<1$, we can proceed in a similar manner to the above and deform the contour to an integral over the imaginary axis. This gives

$$
\tilde{\zeta}(s)=\frac{\sin \pi s}{\pi} \int_{\lambda}^{\infty} d x\left(x^{2}-\lambda^{2}\right)^{-s} \frac{d}{d x} \ln |F(i x)| .
$$

We again add and subtract the terms that cause the integral to diverge at $s=0$. We find that the analytic continuation of Eq. (28) at $s=0$ is

$$
\begin{aligned}
\tilde{\zeta}(0) & =\frac{\chi}{2}, \\
\tilde{\zeta}^{\prime}(0) & =-\ln |\pi F(i \lambda)| .
\end{aligned}
$$

Hence, we can now write down the complete $\zeta$ function and its derivative at $s=0$. We find

$$
\begin{aligned}
\zeta(0)= & \frac{1}{16 \pi^{2} a^{4}} \int\left|g^{(4)}\right| d^{4} x\left\{-\chi \frac{17}{960}-\sum_{i=1,2}\left(r_{4}\left(\theta_{i}\right)+\frac{1}{4} r_{2}\left(\theta_{i}\right)\right)\right\}, \\
\zeta^{\prime}(0)= & -\frac{a^{-4}}{8 \pi^{2}} \int\left|g^{(4)}\right| d^{4} x\left(G\left(\theta_{1}, \theta_{2}\right)+C_{1}\left(\theta_{1}\right)+C_{2}\left(\theta_{2}\right)+q\right) \\
& +\ln a^{2} \zeta(0)-2 \gamma \zeta(0),
\end{aligned}
$$

where the "non-local" part in Eq. (33) is

$$
\begin{aligned}
G\left(\theta_{1}, \theta_{2}\right)= & \int_{0}^{\infty} d x x\left(x^{2}+\frac{1}{4}\right) \ln \left|\frac{F(i x)}{F_{a}(i x)}\right| \\
& -2 \int_{0}^{\infty} d x x\left(x^{2}+\frac{1}{4}\right)\left(1+e^{2 \pi x}\right)^{-1} \ln |\pi F(i x)|
\end{aligned}
$$


the "local" functions $C_{1}(\theta)$ and $C_{2}(\theta)$ are

$$
\begin{aligned}
C_{1}(\theta)= & \int_{0}^{\infty} d x x^{3}\left\{\ln \left|\Sigma^{S}(x, \theta)\right|-\sum_{k=1}^{3} s_{k}(\theta) x^{-k}-s_{4}(\theta) x^{-4} e^{-1 / x}\right\} \\
& +\frac{1}{4} \int_{0}^{\infty} d x x\left\{\ln \left|\Sigma^{S}(x, \theta)\right|-s_{1}(\theta) x^{-1}-s_{2}(\theta) x^{-2} e^{-1 / x}\right\}+\frac{1}{2} s_{4}(\theta), \\
C_{2}(\theta)= & \int_{0}^{\infty} d x x^{3}\left\{\ln \left|\Sigma^{R}(x, \theta)\right|-\sum_{k=1}^{3} r_{k}(\theta) x^{-n}-r_{4}(\theta) x^{-4} e^{-1 / x}\right\} \\
& +\frac{1}{4} \int_{0}^{\infty} d x x\left\{\ln \left|\Sigma^{R}(x, \theta)\right|-r_{1}(\theta) x^{-1}-r_{2}(\theta) x^{-2} e^{-1 / x}\right\}+\frac{1}{2} r_{4}(\theta),
\end{aligned}
$$

and the constant part is

$$
q=\chi \gamma \frac{17}{960}+\int_{0}^{\infty} d x x\left(x^{2}+\frac{1}{4}\right) \ln \left|\frac{\sinh \pi x}{\cosh \pi(x-i \mu)}\right|
$$

The one-loop effective action in the conformally rescaled metric is

$$
W_{\Omega}=-\frac{1}{2} \zeta^{\prime}(0)-\frac{1}{2} \zeta(0) \ln \mu_{R}^{2},
$$

so it can be seen that the terms proportional to $\zeta(0)$ in Eq. (33) can be absorbed into a redefinition of the renormalization scale $\mu_{R}$.

As a check on our results, the renormalization scale dependent term $\zeta(0)$ can also be calculated directly using heat kernel methods. This is done in Section IV

\section{COCYCLE FUNCTION}

The cocycle function for the conformal rescaling was given in Eq. (10) in terms of the $B_{5 / 2}(f, \Delta)$ heat kernel coefficient. The $B_{5 / 2}(f, \Delta)$ heat kernel coefficient has been calculated for general operators of Laplace type with mixed Dirichlet and Robin boundary conditions in 33. It is comprised of curvature terms of order $R^{2}$ evaluated only on the boundary of the spacetime. The general expression is quite lengthy. However, for a scalar field obeying Dirichlet boundary conditions, many terms are zero. Additionally, the heat kernel coefficients simplify further for the case of a maximally symmetric boundary.

The calculation of the heat kernel coefficient is straightforward but tedious. For the metric (10) and operator (4), we find that the cocycle function can be written as

$$
C[\Omega]=\sum_{i=1,2} \frac{1}{a^{4}(4 \pi)^{2}} \int\left|g^{(4)}\right|^{1 / 2} d^{4} x\left\{\omega\left(z_{i}\right) \mathcal{A}_{i}+\mathcal{B}_{i}\right\},
$$

where

$$
\mathcal{A}_{i}=\frac{17}{1920}+\frac{3}{16} \frac{1+\cos ^{2} \theta_{i}}{\sin ^{4} \theta_{i}}\left(\mu^{2}-1 / 4\right)-\frac{1}{8} \frac{1}{\sin ^{4} \theta_{i}}\left(\mu^{2}-1 / 4\right)^{2},
$$

and

$$
\mathcal{B}_{i}=-\frac{35}{192}-\frac{889}{1024} \cot ^{4} \theta_{i}-\frac{265}{256} \cot ^{2} \theta_{i}-\frac{1}{64} \frac{\mu^{2}-4}{\sin ^{4} \theta_{i}}\left(17 \cos ^{2} \theta_{i}+4\right),
$$

where we have reintroduced $\mu=\sqrt{4-20 \xi+m^{2} / \sigma^{2}}$.

One can also use the heat kernel to directly evaluate $\zeta(0)$ through the relation

$$
\zeta(0)=B_{5 / 2}(1, \Delta)=\sum_{i=1,2} \frac{1}{a^{4}(4 \pi)^{2}} \int\left|g^{(4)}\right|^{1 / 2} d^{4} x \mathcal{A}_{i} .
$$


Inserting the expressions for $r_{2}(\theta)$ and $r_{4}(\theta)$ from Appendix B into the expression for $\zeta(0)$ for the conformally transformed operator (32), it is easily seen that there is exact agreement between the two calculations. These expressions also reduce to previously calculated values for flat branes [17, 18] in the limit $a \rightarrow \infty$.

It is interesting to notice that the heat kernel coefficient $B_{5 / 2}$ is comprised entirely of local geometrical objects, while the full $\zeta$ function is a nonlocal quantity as information from both boundaries is required for the eigenvalue problem. It is therefore quite remarkable that $\zeta(0)$ can be obtained from the heat kernel coefficient, and this is a powerful check on our method.

\section{MASSLESS CONFORMALLY COUPLED CASE}

The integrals in equations (34) and (35) cannot be done analytically in the general case we must resort to numerical methods. However, in the case $m=0$, and $\xi=3 / 16$, we find $\mu=1 / 2$ and the Legendre functions simplify to hyperbolic functions and exponentials, so that

$$
R_{-1 / 2+i x}^{-1 / 2}(\theta)=\frac{1}{x} \sqrt{\frac{2}{\pi}} \sinh (x \theta)
$$

and

$$
S_{-1 / 2+i x}^{1 / 2}(\theta)=\sqrt{\frac{2}{\pi}} e^{-x \theta}
$$

All the coefficients $r_{k}(\theta)$ and $s_{k}(\theta)$ vanish. The local functions $C_{1}(\theta)$ and $C_{2}(\theta)$ also vanish. The constant $q$ can be absorbed into a redefinition of the renormalization scale $\mu_{R}$. The expression for $\zeta(0)$ also becomes simple. We find

$$
\zeta(0)=\frac{1}{16 \pi^{2} a^{4}} \int\left|g^{(4)}\right|^{1 / 2} d^{4} x \frac{17}{960} .
$$

The integral in the first term for the nonlocal part $G\left(\theta_{1}, \theta_{2}\right)$ can be done explicitly in terms of the Riemann zeta function $\zeta_{R}(s)$. The remaining integral in $G\left(\theta_{1}, \theta_{2}\right)$ must be none numerically. We find

$$
\begin{aligned}
V_{\Omega}=\frac{1}{16 \pi^{2} a^{4}} & \left\{-\frac{3 \zeta_{R}(5)}{8 L^{4}}-\frac{\zeta_{R}(3)}{16 L^{2}}-2 \int_{0}^{\infty} d x \frac{x\left(x^{2}+\frac{1}{4}\right)}{e^{2 \pi x}+1} \ln \frac{2 \sinh L x}{x}\right. \\
& \left.-\frac{17}{1920} \ln \left(a^{2} \mu_{R}^{2}\right)\right\},
\end{aligned}
$$

where $L=\theta_{2}-\theta_{1}=z_{2} / a-z_{1} / a$. From the previous equation, it is clear that the effective potential in the conformally rescaled metric is a function solely of the nondimensional conformal distance $L$.

We plot the effective potential $V_{\Omega}$ as a function of $L$ in Figure 1 We have adjusted the renormalisation scale so that the maximum of the potential is at $V_{\Omega}=0$.

To this result, we must also add the cocycle function, discussed in Section IV The total effective potential is then

$$
V=V_{\Omega}+\sum_{i=1,2} \frac{1}{16 \pi^{2} a^{4}}\left\{\frac{17}{1920} \ln \sigma a \sin \theta_{i}+\frac{5}{96}+\frac{25}{128} \cot ^{2} \theta_{i}+\frac{131}{1024} \cot ^{4} \theta_{i}\right\} .
$$

The total effective potential $V$ is now no longer a function of $L=\theta_{1}-\theta_{2}$, but now depends explicitly on $\theta_{1}$ and $\theta_{2}$. The terms from the cocycle function can dominate the effective potential if $\theta_{1}$ or $\theta_{2}$ is small or close to $\pi$. Also, the terms due to the cocycle function cannot be absorbed into a redefinition of the brane tensions as is often done in the case of flat branes.

It is worthwhile mentioning here that one cannot obtain the effective action for a conformally coupled scalar field in the background with de Sitter branes, obtained in 21, 22], by a simple analytic continuation of $a \rightarrow i a$. This is because of the different global properties of $S^{4}$ and $\mathrm{H}^{4}$, as has been noted in previous calculations of $\zeta$ functions on $S^{4}$ and $\mathrm{H}^{4}[28]$. 


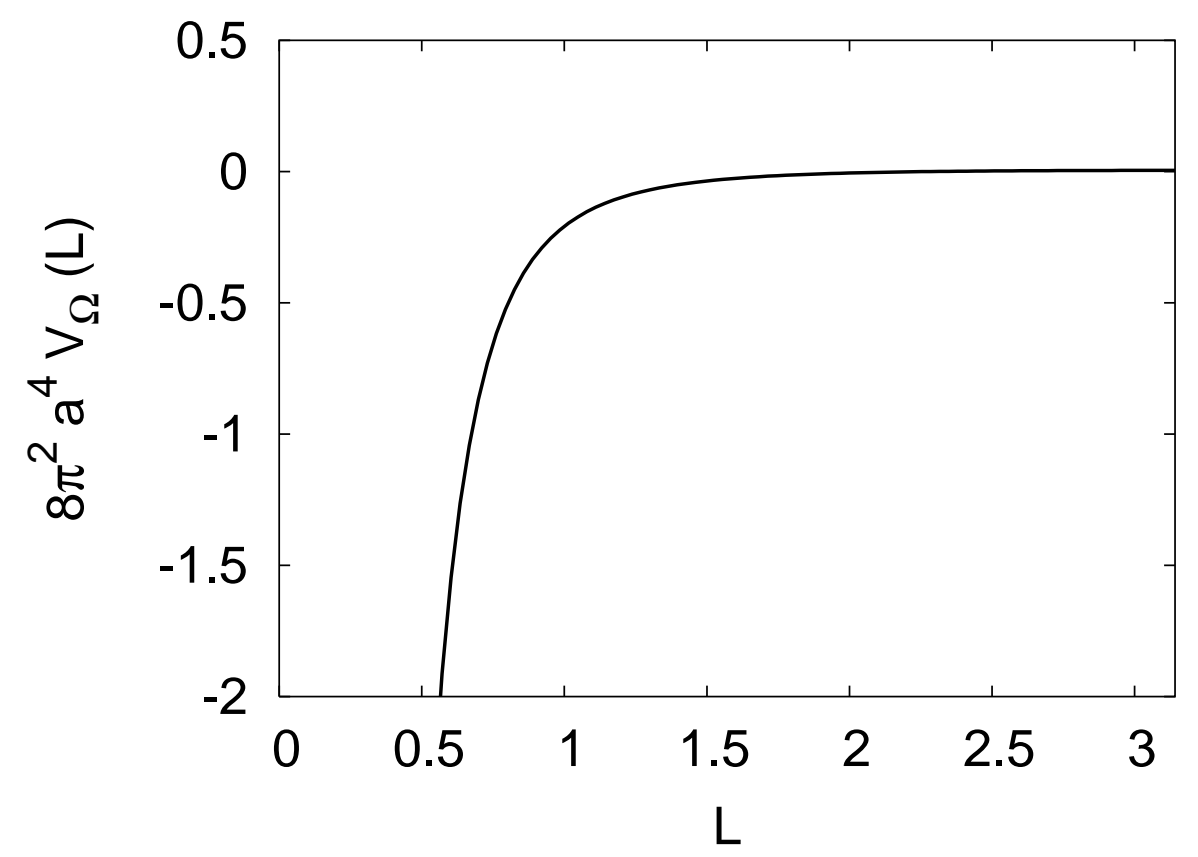

FIG. 1: Numerical results for the effective potential due to a conformally coupled scalar field as a function of the non-dimensional conformal length $L=\theta_{2}-\theta_{1}=z_{2} / a-z_{1} / a$, where $z_{1}$ and $z_{2}$ are the positions of the branes in conformal coordinate and $a$ is the $A d S_{4}$ radius of the brane at $z_{1}$

\section{MORE GENERAL CASES}

\section{A. Numerical Results}

In the case of general mass and coupling constant $\xi$, the integrals in equations (34), (35) and (37) must be computed numerically. As an example, we have chosen $m=0$ and $\xi=3 / 20$, so that $\mu=1$. The integrals that must be evaluated are fairly computationally intensive, but can be performed simply (if slowly) using computer packages such as MAPLE or MATHEMATICA.

The numerical results for the nonlocal part of the effective potential $G\left(\theta_{1}, \theta_{2}\right.$ are shown as a function of $\theta_{1}=z_{1} / a$ and $\theta_{2}=z_{2} / a$ in Figure 2 It can be seen from this figure that this part of the effective action is not solely dependent on the difference $\theta_{2}-\theta_{1}$, unlike the conformally coupled case considered above. Also, this term in the effective action diverges strongly to negative values as $\theta_{2}-\theta_{1} \rightarrow 0$.

The local functions $C_{1}(\theta)$ and $C_{2}(\theta)$ are shown in Figure 3] These terms can become significant if $\theta_{1}$ or $\theta_{2}$ are small or close to $\pi$. Finally, We calculate the constant $q$ to be

$$
q \approx-0.03128 .
$$

These numerical results enable one to calculate the finite part of the effective action of the conformally rescaled operator. However, to this, we must also add the renormalization scale dependent term $\zeta(0) \ln \mu_{R}^{2}$. From both Eq. (32) and the $B_{5 / 2}(1, \Delta)$ heat kernel coefficient, one can calculate the value of $\zeta(0)$. Using either method, we find

$$
\zeta(0)=\frac{1}{16 \pi^{2} a^{4}} \sum_{i=1,2} \int\left|g^{(4)}\right|^{1 / 2} d^{4} x\left\{\frac{19}{240}+\frac{9}{32} \cot ^{2} \theta_{i}+\frac{27}{128} \cot ^{4} \theta_{i}\right\} .
$$

We have now calculated all the terms in the effective potential in the conformally rescaled metric $V_{\Omega}$. The total effective potential including the cocycle function is then

$$
\begin{aligned}
V=V_{\Omega}+\sum_{i=1,2} \frac{1}{16 \pi^{2} a^{4}} & \left\{\left(\frac{19}{240}+\frac{9}{32} \cot ^{2} \theta_{i}+\frac{27}{128} \cot ^{4} \theta_{i}\right) \ln \sigma a \sin \theta_{i}\right. \\
& \left.+\frac{1}{192}-\frac{13}{256} \cot ^{2} \theta_{i}-\frac{73}{1024} \cot ^{4} \theta_{i}\right\} .
\end{aligned}
$$




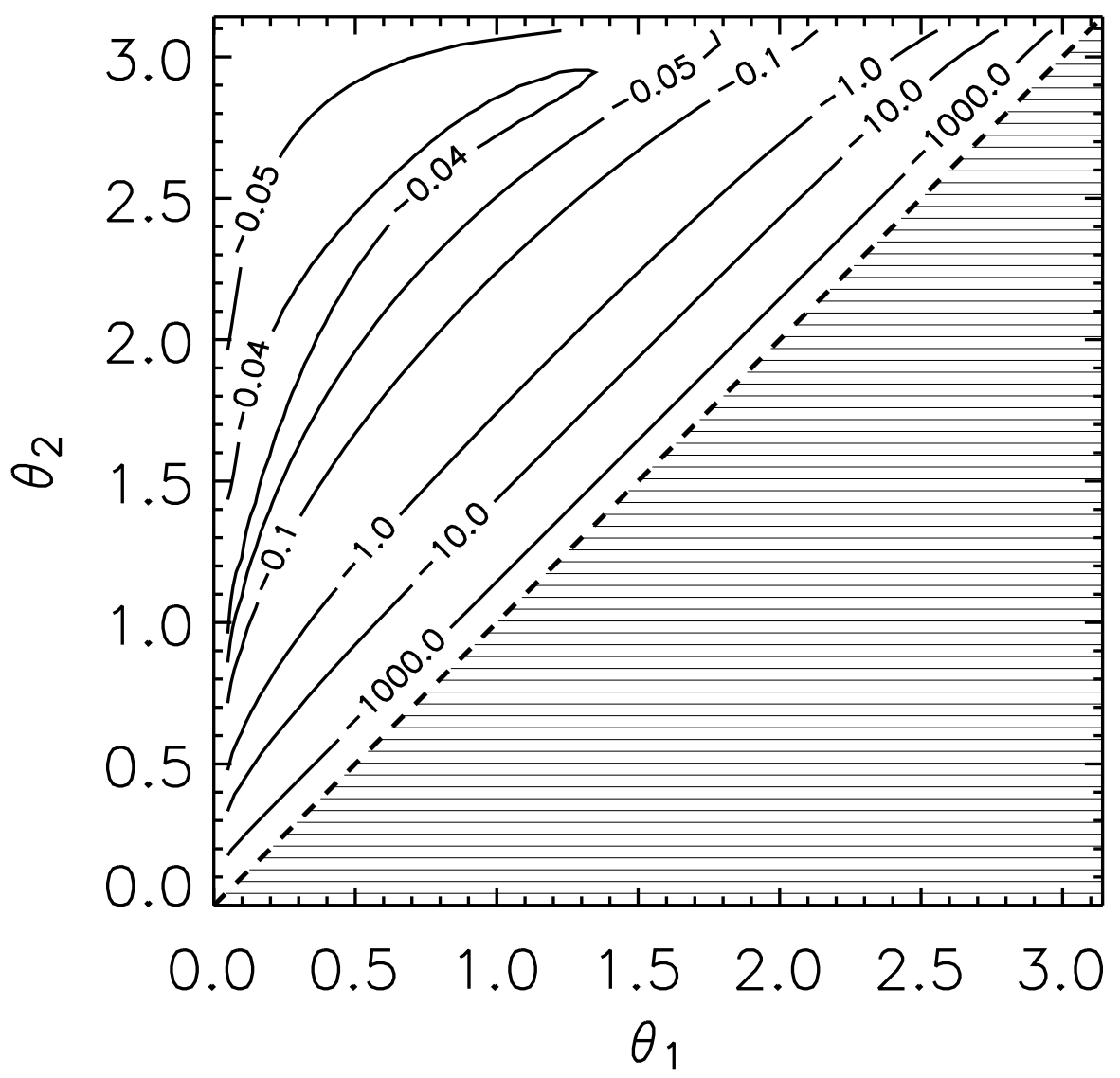

FIG. 2: Numerical results for the non-local part of the effective action. $\theta_{1}$ and $\theta_{2}$ are $z_{1} / a$ and $z_{2} / a$ respectively, where $z_{1}$ and $z_{2}$ are the positions of the branes in the conformal coordinate $z$, and $a$ is the $A d S_{4}$ radius of the brane at $z_{1}$. The contours are values of $G\left(\theta_{1}, \theta_{2}\right)$. The shaded region is disallowed, since we have restricted $z_{2}>z_{1}$

\section{B. Analytical approximation}

The numerical results in Section $\mathbf{\nabla I A}$ show that the effective potential becomes very large if $L=\theta_{2}-\theta_{2} \ll 1$. In this case, the dominating contribution to the effective potential is from the integral in the first term in Eq. (34). We can approximate this integral using the asymptotic expansion of the Legendre functions. This gives a series in powers of $L$. The first two terms in this expansion of the effective potential are

$$
V \approx-\frac{3 \zeta_{R}(5)}{128 \pi^{2}\left(z_{2}-z_{1}\right)^{4}}+\frac{\zeta_{R}(3)}{64 \pi^{2}\left(z_{2}-z_{1}\right)^{2}}\left[\sigma^{2}\left(\mu^{2}-1 / 4\right)-\frac{1}{4 a^{2}}\right]+O\left(\ln \left(z_{2}-z_{1}\right)\right.
$$

The first term on the right hand side of Eq. (51) is the Casimir potential for two flat branes in flat space, separated by a distance $z_{2}-z_{1}$. Note that this leading term is independent of the mass or coupling to the curvature of the scalar field. A similar result was found for the small distance approximation with flat branes in 17].

The second term in Eq. (51) is the first order correction to this result due to bulk and brane curvature. This approximation results in a much more manageable expression than the exact expression for the effective potential.

\section{CONCLUSIONS}

We have calculated the one-loop effective potential for a scalar field with general mass and coupling to the Ricci scalar in the two brane Randall-Sundrum model with detuned brane tensions such that the boundary branes are 


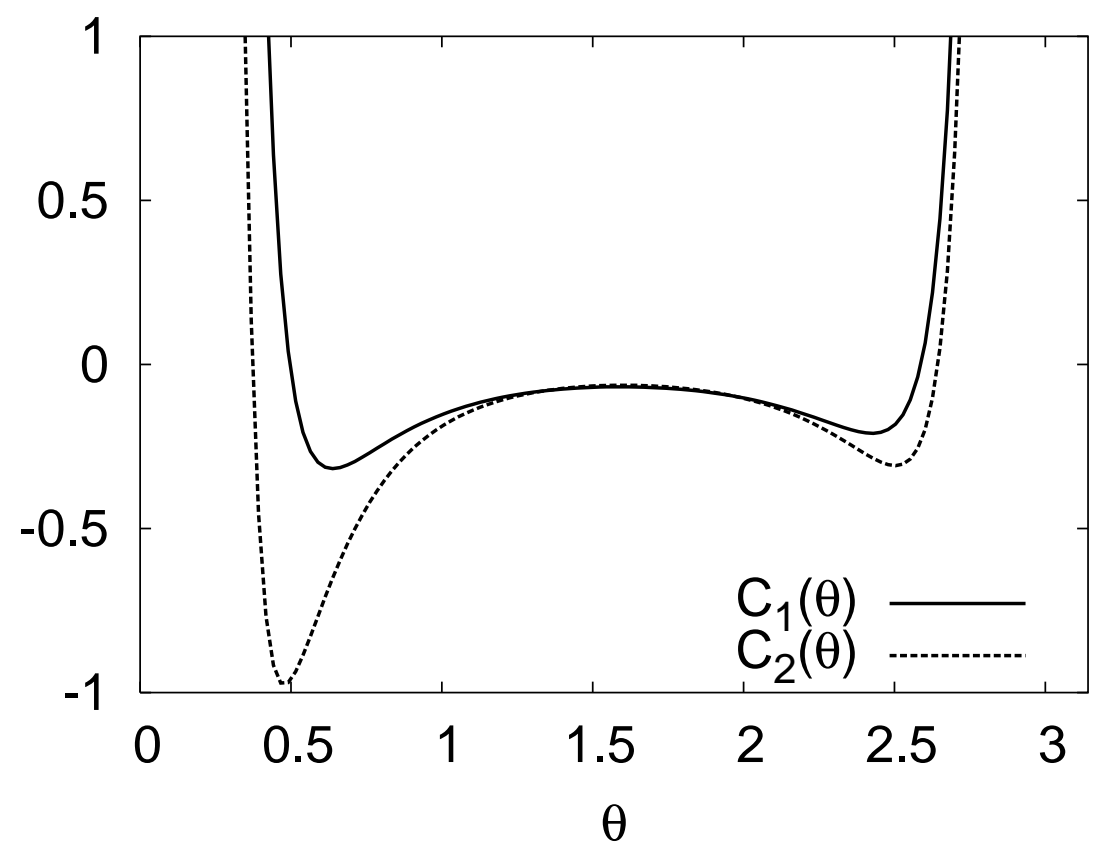

FIG. 3: Numerical results for the local functions $C_{1}(\theta)$ and $C_{2}(\theta)$ in the effective potential. Again, $\theta_{1}=z_{1} / a$ and $\theta_{2}=z_{2} / a$ where $z_{1}$ and $z_{2}$ are the positions of the branes in conformal coordinates, and $a$ is the $A d S_{4}$ radius of the brane at $z_{1}$.

$A d S_{4}$. Conformal rescalings of the metric are used to relate the metric to two hyperbolic branes. In general, the resulting expressions contain integrals of Legendre functions which must be performed numerically. We obtain some approximations in the small conformal distance limit, which reduce to the Casimir potential for two flat branes in flat space, and also calculate the first order corrections due to the brane curvature. Our results are checked by comparing the renormalization scale dependence obtained from the conformally rescaled operator with a direct computation using heat kernel coefficients. They are found to agree exactly.

It should be remembered that, in the dimensionally reduced theory, there is also a classical potential for the radion field, unless the brane tensions are tuned. It is interesting to compare the form of the classical radion potential and the quantum effective potential for the radion due to a bulk scalar field. The classical radion effective potential has been analyzed in [19]. The classical potential is most naturally expressed in terms of the proper distance $r$, related to $z_{1}$ and $z_{2}$ by

$$
\pi r=\int_{z 1}^{z 2} e^{-w(z)} d z=\frac{1}{\sigma} \ln \tan \frac{z_{2}}{2 a}-\frac{1}{\sigma} \ln \tan \frac{z_{1}}{2 a}
$$

For small conformal separation, $\pi r \approx z_{2}-z_{1}$ and the classical effective potential goes like

$$
V_{\text {classical }} \approx \operatorname{const}(\sigma a \pi r)^{-2} \approx \operatorname{const} \frac{1}{\left(\sigma a\left(z_{2}-z_{1}\right)\right)^{2}}
$$

where "const" is a positive constant. One can see from Eq. (51) that the effective potential generated by a quantized bulk scalar field would destabilize the classical potential at small separation, as the quantum effective potential diverges faster than the classical potential in this limit. Of course, there will also be an effective potential generated by graviton fluctuations which have not been included in this discussion.

It would be interesting to extend this calculation to higher spin fields so that one could investigate the effect of the supersymmetry breaking on the Casimir potential. An intriguing possibility in the supersymmetric Randall-Sundrum model is that one-loop effects could generate a potential for the supersymmetry breaking parameter (either the twist angle of the gravitino boundary condition or the v.e.v. of the fifth component of the gauge field) which is a modulus of compactification at the classical level [34]. This situation is reminiscent of supersymmetry breaking in heterotic $\mathrm{M}$ theory by gaugino condensation [35]. 


\section{Acknowledgments}

I thank Ian Moss for helpful discussions and comments on an earlier draft of this paper.

\section{APPENDIX A: ROBIN BOUNDARY CONDITIONS}

\section{1. $\zeta$-function on $\mathrm{H}^{4} \times I$}

Robin boundary conditions have some combination of the field and its normal derivative vanishing on the boundary. We take

$$
\left(\partial_{N}+\eta K\right) \phi=0 \quad \text { on } \quad \partial \mathcal{M} .
$$

where $K$ is the trace of the extrinsic curvature of the boundary and $N$ denotes the outward pointing unit normal. Under a conformal transformation, this changes to

$$
\left(\partial_{N}+\hat{\eta} K \Omega^{-1}\right) \tilde{\phi}=0 \quad \text { on } \partial \mathcal{M},
$$

where $\hat{\eta}=\eta-3 / 8$ since the field $\phi$ also transforms under the conformal rescaling. Applying this boundary condition to the general solution, the implicit equation for the $b_{n}$ 's can be given in terms of new functions $T_{\nu}^{-\mu}(\theta)$ and $U_{\nu}^{\mu}(\theta)$, defined by

$$
\begin{aligned}
T_{\nu}^{-\mu}(\theta) & =R_{\nu}^{-\mu+1}(\theta)+\cot \theta(1 / 2-\mu-4 \hat{\eta}) R_{\nu}^{-\mu}(\theta), \\
U_{\nu}^{\mu}(\theta) & =S_{\nu}^{\mu+1}(\theta)+\cot \theta(1 / 2+\mu-4 \hat{\eta}) S_{\nu}^{\mu}(\theta),
\end{aligned}
$$

as

$$
F\left(b_{n}\right)=T_{-1 / 2+b_{n}}^{-\mu}\left(\theta_{1}\right) U_{-1 / 2+b_{n}}^{\mu}\left(\theta_{2}\right)-T_{-1 / 2+b_{n}}^{-\mu}\left(\theta_{2}\right) U_{-1 / 2+b_{n}}^{\mu}\left(\theta_{1}\right)=0 .
$$

The procedure then follows the Dirichlet case, in that we write the $\zeta$ function as a contour integral, shift the contour to the real axis, and then analytically continue to find the effective action. If we define the functions $\Sigma^{T}(x, \theta)$ and $\Sigma^{U}(x, \theta)$ by

$$
T_{-1 / 2+i x}^{-\mu}(\theta)=e^{i \pi \mu / 2+i \pi / 4} \frac{x}{\sqrt{2 \pi}} e^{x \theta} \frac{\Gamma(i x-\mu+1 / 2)}{\Gamma(i x+1)} \Sigma^{T}(x, \theta),
$$

and

$$
U_{-1 / 2+i x}^{\mu}(\theta)=-e^{i \pi \mu / 2-i \pi / 4} x \sqrt{\frac{2}{\pi}} e^{-x \theta} \frac{\Gamma(i x+\mu+1 / 2)}{\Gamma(i x+1)} \Sigma^{U}(x, \theta),
$$

then $\Sigma^{T}(x, \theta)$ and $\Sigma^{U}(x, \theta)$ have asymptotic expansions of the Poincaré type. Similar to the Dirichlet case, we define coefficients $t_{k}(\theta)$ and $u_{k}(\theta)$ by

$$
\ln \left|\Sigma^{T}(x, \theta)\right| \sim \sum_{k=1}^{\infty} t_{k}(\theta) x^{-k}+O\left(e^{-x}\right), \quad \ln \left|\Sigma^{U}(x, \theta)\right| \sim \sum_{k=1}^{\infty} u_{k}(\theta) x^{-k} .
$$

We again refer the reader to Appendix $\mathbb{B}$ for explicit expressions for $t_{k}(\theta)$ and $u_{k}(\theta)$.

Thus, for large $x$,

$$
\ln |F(i x)| \sim\left(\theta_{2}-\theta_{1}\right) x+\chi \ln (x)-\ln \pi+O\left(x^{-2}\right)
$$

where now

$$
\chi=1 .
$$

The effective action in the conformally rescaled metric for Robin boundary conditions can now be found by substituting $R, S, \Sigma^{R}, \Sigma^{S}, r_{k}$ and $s_{k}$ in the Dirichlet case by $T, U, \Sigma^{T}, \Sigma^{U}, t_{k}$ and $u_{k}$. Additionally, one must replace $\chi=-1$ for Dirichlet boundary conditions by $\chi=+1$ for Robin boundary conditions.

One subtlety in the Robin case is that there may exist zero or imaginary solutions of $F\left(b_{n}\right)=0$. For example, a conformally coupled scalar field with Robin boundary conditions has a zero mode. This means that there will be a contribution to the $\zeta$-function from integral over the small semi-circle $\mathcal{C}_{\varepsilon}$ as $\varepsilon \rightarrow 0$. Imaginary values of $b_{n}$ signal an instability, and these situations should be physically unacceptable. In our analysis, we assume that the values of $\eta, \xi$ and $m$ are such that there are no zero or imaginary solutions of $F\left(b_{n}\right)=0$. 


\section{Cocycle function}

The heat kernel coefficient for scalar fields obeying Robin boundary conditions is a little more lengthy than the Dirichlet case. Again, the calculation is straightforward, but messy. For the cocycle function, we find that Eqs. (40) and (41) become

$$
\begin{aligned}
\mathcal{A}_{i}= & -\frac{17}{1920}+64 \cot ^{4} \theta_{i} \hat{\eta}^{4}+2 \cot ^{2} \theta_{i} \hat{\eta}^{2}+\frac{1}{8} \frac{1}{\sin ^{4} \theta_{i}}\left(\mu^{2}-1 / 4\right)^{2} \\
& -\frac{1}{\sin ^{4} \theta_{i}}\left[\cos ^{2} \theta_{i}\left(8 \hat{\eta}^{2}+2 \hat{\eta}\right)+\frac{3}{16}\left(\cos ^{2} \theta_{i}+1\right)\right]\left(\mu^{2}-1 / 4\right)
\end{aligned}
$$

and

$$
\begin{aligned}
\mathcal{B}_{i}= & \left(\frac{61}{15360}+\frac{11}{48} \hat{\eta}-\frac{2}{3} \hat{\eta}^{2}-8 \hat{\eta}^{3}\right) \cot ^{4} \theta_{i}+\left(\frac{623}{768}+\frac{71}{24} \hat{\eta}-\hat{\eta}^{2}\right) \frac{\cos ^{2} \theta_{i}}{\sin ^{4} \theta_{i}} \\
& +\frac{35}{192} \frac{1}{\sin ^{4} \theta_{i}}+\left[\left(\hat{\eta}+\frac{15}{64}\right) \cos ^{2} \theta_{i}+\frac{1}{16}\right] \frac{\mu^{2}-4}{\sin ^{4} \theta_{i}}
\end{aligned}
$$

respectively.

Again, these results reduce to the previously known flat brane values calculated in [18] in the limit $a \rightarrow \infty$.

\section{An example}

In some special cases, the implicit equation for the eigenvalues for Robin boundary conditions can be reduced to the one resembling that for a Dirichlet case, plus one "extra" mode. As an example we consider the case where $\mu=0$ and $\eta=1 / 2$. Then the implicit equation for the $b_{n}$ reduces to

$$
\left(b_{n}^{2}-\frac{1}{4}\right)\left(R_{-1 / 2+b_{n}}^{-1}\left(\theta_{1}\right) S_{-1 / 2+b_{n}}^{1}\left(\theta_{2}\right)-R_{-1 / 2+b_{n}}^{-1}\left(\theta_{2}\right) S_{-1 / 2+b_{n}}^{1}\left(\theta_{1}\right)\right)=0 .
$$

This is simply the example considered in Section VI but with the "extra" mode at $b_{n}=1 / 2$. We can add this extra mode to the $\zeta$-function considered in Section VI by hand. This gives an extra contribution to $\zeta(0)$ of $-\left(240 \pi^{2} a^{4}\right)^{-1}$, giving

$$
\zeta(0)=\frac{1}{16 \pi^{2} a^{4}} \sum_{i=1,2} \int\left|g^{(4)}\right|^{1 / 2} d^{4} x\left\{\frac{11}{240}+\frac{9}{32} \cot ^{2} \theta_{i}+\frac{27}{128} \cot ^{4} \theta_{i}\right\}
$$

again in agreement with the value calculated directly from the heat kernel coefficient $B_{5 / 2}(1, \Delta)$. The extra mode will also give an extra contribution to $\zeta^{\prime}(0)$ of a constant (independent of $\theta_{1}$ or $\theta_{2}$ ).

However, the cocycle function cannot be deduced from the Dirichlet case, and must be computed explicitly.

\section{APPENDIX B: COEFFICIENTS IN ASYMPTOTIC EXPANSIONS OF THE LEGENDRE FUNCTIONS}

\section{Dirichlet boundary conditions}

From the asymptotic expansion of the Legendre functions for large degree [31, page 146], the asymptotic expansion of $\ln \left|\Sigma^{R}(x, \theta)\right|$ and $\ln \left|\Sigma^{S}(x, \theta)\right|$ can be shown to be of the form

$$
\ln \left|\Sigma^{R}(x, \theta)\right| \sim \sum_{k=1}^{\infty} r_{k}(\theta) x^{-k}, \quad \ln \left|\Sigma^{S}(x, \theta)\right| \sim \sum_{k=1}^{\infty} s_{k}(\theta) x^{-k} .
$$


The coefficients are easily evaluated with the help of a computer algebra package such as MAPLE or MATHEMATICA. We obtain the first four coefficients as

$$
\begin{aligned}
& r_{1}(\theta)=-\frac{1}{2} \cot \theta\left(\mu^{2}-1 / 4\right), \\
& r_{2}(\theta)=-\frac{1}{4} \frac{1}{\sin ^{2} \theta}\left(\mu^{2}-1 / 4\right), \\
& r_{3}(\theta)=-\frac{1}{96} \frac{\cos \theta}{\sin ^{3} \theta}\left(\mu^{2}-1 / 4\right)\left(8 \mu^{2} \cos ^{2} \theta-12 \mu^{2}-2 \cos ^{2} \theta+27\right), \\
& r_{4}(\theta)=\frac{1}{32} \frac{1}{\sin ^{4} \theta}\left(\mu^{2}-1 / 4\right)\left(4 \mu^{2}-8 \cos ^{2} \theta-5\right),
\end{aligned}
$$

and $s_{n}(\theta)=(-1)^{n} r_{n}(\theta)$.

\section{Robin boundary conditions}

Similarly to the Dirichlet case, for Robin boundary conditions we have

$$
\ln \left|\Sigma^{T}(x, \theta)\right| \sim \sum_{k=1}^{\infty} t_{k}(\theta) x^{-k}, \quad \ln \left|\Sigma^{U}(x, \theta)\right| \sim \sum_{k=1}^{\infty} u_{k}(\theta) x^{-k} .
$$

This time, we find

$$
\begin{aligned}
& t_{1}(\theta)=-\frac{1}{2} \cot \theta\left(\mu^{2}-1 / 4+8 \hat{\eta}\right) \\
& t_{2}(\theta)=\frac{1}{4 \sin ^{2} \theta}\left(\mu^{2}-1 / 4-32 \hat{\eta}^{2} \cos ^{2} \theta\right) \\
& t_{3}(\theta)=-\frac{1}{384} \frac{\cos \theta}{\sin ^{3} \theta}\left(21-72 \mu^{2}-48 \mu^{4}+2 \cos ^{2} \theta-16 \mu^{2} \cos ^{2} \theta\right. \\
&\left.\quad+32 \mu^{4} \cos ^{2} \theta+192 \hat{\eta}+8192 \hat{\eta}^{3} \cos ^{2} \theta-768 \mu^{2} \hat{\eta}\right) \\
& t_{4}(\theta)=-\frac{1}{128 \sin ^{4} \theta}\left(5-24 \mu^{2}+16 \mu^{4}+8 \cos ^{2} \theta+8192 \hat{\eta}^{4} \cos ^{4} \theta-32 \mu^{2} \cos ^{2} \theta\right. \\
&\left.\quad+256 \hat{\eta}^{2} \cos ^{2} \theta+64 \hat{\eta} \cos ^{2} \theta-256 \mu^{2} \hat{\eta} \cos ^{2} \theta-1024 \mu^{2} \hat{\eta}^{2} \cos ^{2} \theta\right)
\end{aligned}
$$

and $u_{i}(\theta)=(-1)^{i} t_{i}(\theta)$.

[1] Lisa Randall and Raman Sundrum. A large mass hierarchy from a small extra dimension. Phys. Rev. Lett., 83:3370-3373, 1999.

[2] Lisa Randall and Raman Sundrum. An alternative to compactification. Phys. Rev. Lett., 83:4690-4693, 1999.

[3] Nemanja Kaloper. Bent domain walls as braneworlds. Phys. Rev., D60:123506, 1999.

[4] Andreas Karch and Lisa Randall. Locally localized gravity. JHEP, 05:008, 2001.

[5] Yoshiaki Himemoto and Misao Sasaki. Brane-world inflation without inflaton on the brane. Phys. Rev., D63:044015, 2001.

[6] Philippe Brax and Zygmunt Lalak. Brane world supersymmetry, detuning, flipping and orbifolding. Acta Phys. Polon., B33:2399-2418, 2002.

[7] Jonathan Bagger and Dmitry V. Belyaev. Supersymmetric branes with (almost) arbitrary tensions. Phys. Rev., D67:025004, 2003.

[8] Jonathan Bagger and Dmitry Belyaev. Twisting warped supergravity. JHEP, 06:013, 2003.

[9] Walter D. Goldberger and Ira Z. Rothstein. Quantum stabilization of compactified ads(5). Phys. Lett., B491:339-344, 2000.

[10] Jaume Garriga, Oriol Pujolas, and Takahiro Tanaka. Radion effective potential in the brane-world. Nucl. Phys., B605:192214, 2001.

[11] A. Flachi and D. J. Toms. Quantized bulk scalar fields in the randall-sundrum brane- model. Nucl. Phys., B610:144-168, 2001.

[12] Ralf Hofmann, Panagiota Kanti, and Maxim Pospelov. (de-)stabilization of an extra dimension due to a casimir force. Phys. Rev., D63:124020, 2001. 
[13] A. A. Saharian and M. R. Setare. The casimir effect on background of conformally flat brane- world geometries. Phys. Lett., B552:119-126, 2003.

[14] A. Knapman and D. J. Toms. Stress-energy tensor for a quantised bulk scalar field in the randall-sundrum brane model. Phys. Rev., D69:044023, 2004.

[15] Antonino Flachi, Ian G. Moss, and David J. Toms. Quantized bulk fermions in the randall-sundrum brane model. Phys. Rev., D64:105029, 2001.

[16] Antonino Flachi, Ian G. Moss, and David J. Toms. Fermion vacuum energies in brane world models. Phys. Lett., B518:153156, 2001.

[17] Jaume Garriga, Oriol Pujolas, and Takahiro Tanaka. Moduli effective potential in warped-brane world compactifications. Nucl. Phys., B655:127-169, 2003.

[18] Ian G. Moss and James P. Norman. One loop effective potential in heterotic m-theory. 2004.

[19] Jonathan Bagger and Michele Redi. Radion effective theory in the detuned randall-sundrum model. 2003.

[20] Wade Naylor and Misao Sasaki. Casimir energy for de sitter branes in bulk ads(5). Phys. Lett., B542:289-294, 2002.

[21] Ian G. Moss, Wade Naylor, Wenceslao Santiago-German, and Misao Sasaki. Bulk quantum effects for de sitter branes in ads(5). Phys. Rev., D67:125010, 2003.

[22] Emilio Elizalde, Shin'ichi Nojiri, Sergi D. Odintsov, and Sachiko Ogushi. Casimir effect in de sitter and anti-de sitter braneworlds. Phys. Rev., D67:063515, 2003.

[23] G. von Gersdorff, M. Quiros, and A. Riotto. Radiative scherk-schwarz supersymmetry breaking. Nucl. Phys., B634:90-104, 2002.

[24] Kunihito Uzawa. Dilaton stabilization in (a)ds spacetime with compactified dimensions. Prog. Theor. Phys., 110:457-498, 2003.

[25] Shin'ichi Nojiri, Sergei D. Odintsov, and Sergio Zerbini. Quantum (in)stability of dilatonic ads backgrounds and holographic renormalization group with gravity. Phys. Rev., D62:064006, 2000.

[26] Shin'ichi Nojiri, Sergei D. Odintsov, and Sergio Zerbini. Bulk versus boundary (gravitational casimir) effects in quantum creation of inflationary brane world universe. Class. Quant. Grav., 17:4855-4866, 2000.

[27] J. S. Dowker and J. P. Schofield. Conformal transformations and the effective action in the presence of boundaries. J. Math. Phys., 31:808, 1990.

[28] R. Camporesi. zeta function regularization of one loop effective potentials in anti-de sitter space-time. Phys. Rev., D43:3958-3965, 1991.

[29] E. Elizalde, S. Leseduarte, and A. Romeo. Sum rules for zeros of bessel functions and an application to spherical aharonovbohm quantum bags. J. Phys. A: Math. Gen., pages 2409-2419, 1993.

[30] M. Bordag, E. Elizalde, and K. Kirsten. Heat kernel coefficients of the laplace operator on the d- dimensional ball. J. Math. Phys., 37:895-916, 1996.

[31] Higher Transcendental Functions, Volume 1. McGraw-Hill Book Company, New York (1953).

[32] M Abramowitz and I. A. Stegun. Handbook of Mathematical Functions. Dover Publications, Inc., New York.

[33] Thomas P. Branson, Peter B. Gilkey, Klaus Kirsten, and Dmitri V. Vassilevich. Heat kernel asymptotics with mixed boundary conditions. Nucl. Phys., B563:603-626, 1999.

[34] Jonathan Bagger and Michele Redi. Supersymmetry breaking by wilson lines in ads(5). 2003.

[35] Petr Horava. Gluino condensation in strongly coupled heterotic string theory. Phys. Rev., D54:7561-7569, 1996.

[36] In fact, $\Delta_{I}$ is the Kaluza-Klein mass operator 